\title{
Equation-based modelling for dynamic optimization of district scale energy systems - a scalability study
}

\author{
Gerald Schweiger \\ Institute of Software Technology \\ Graz University of Technology \\ Graz, Austria \\ gerald.schweiger@tugraz.at
}

\author{
Filip Jorissen \\ Dept. of Mech. Engineering \\ $K U$ Leuven \\ Leuven, Belgium \\ filip.jorissen@kuleuven.be
}

\author{
Hakan Runvik \\ Modelon $A B$ \\ Lund, Sweden \\ hakan.runvik@modelon.com
}

\author{
Lieve Helsen \\ Dept. of Mech. Engineering \\ $K U$ Leuven \\ Leuven, Belgium \\ lieve.helsen@kuleuven.be
}

\begin{abstract}
The optimal operation of energy systems is essential for future sustainable energy systems. While previous studies have shown the potential for equation-based modelling languages for the dynamic optimization of various systems, there is a lack of scalability studies. We investigated the scalability of two different Modelica-based tools for dynamic optimization using two use cases. The first case represents a dynamic optimization of the heating system of a multi-zone building. The second case represents a dynamic optimization of a small-scale district heating system. We show that linear scaling is possible for the tested range.
\end{abstract}

Index Terms-dynamic optimization, district energy system, scalability, equation-based modelling

\section{NOMENCLATURE}

$\begin{array}{ll}p & \text { Vector of parameters. } \\ u & \text { Control variable. } \\ x & \text { State variable. } \\ y & \text { Algebraic variable. } \\ D A E & \text { Differential Algebraic Equations } \\ M P C & \text { Model Predictive Control. } \\ N L P & \text { Nonlinear Programming. } \\ O C T & \text { OPTIMICA Compiler Toolkit. } \\ R E S & \text { Renewable Energy Sources. } \\ T A C O & \text { Toolchain for Automated Control and Opti- } \\ & \text { mization. } \\ V A V & \text { Variable Air Volume. }\end{array}$

\section{INTRODUCTION}

A pressing challenge for future energy systems is to design and operation of systems that integrate large shares of intermittent renewable energy while improving overall system efficiency. As intermittent energy sources expand, other parts of the energy system must become more flexible in order to

The reported research has been conducted within the project GameOpSys (867358) funded by the BMVIT and the VLAIO spin-off Fellowship (HBC.2018.2092), funded by the Flanders Innovation and Entrepreneurship Agency (VLAIO). This work emerged from the IBPSA Project 1, an international project conducted under the umbrella of the International Building Performance Simulation Association (IBPSA). Project 1 will develop and demonstrate a BIM/GIS and Modelica Framework for building and community energy system design and operation. match the available energy from renewable energy sources with the demand. There are multiple options for increasing the flexibility of an energy system, including increasing supply and demand flexibility, combining different energy domains, integrating long and short term energy storage technologies, and increasing the capacity of the transmission and distribution power grid as well as the capacity of interconnections to other countries. Recent studies have shown that the (optimal) operation of buildings and district energy systems have great potential to improve the efficiency of existing systems (see e.g. [1], [2]).

Optimization strategies such as optimal control or Model Predictive Control (MPC) are optimizing control inputs with respect to a given objective. ${ }^{1}$ Both methods require a model of the system (e.g. based on a set of ordinary differential equations, a transfer function or a set of Differential Algebraic Equations (DAE)).

General trends and opportunities for MPC in buildings are discussed in [3], [4]. Previous research has shown that MPC of HVAC systems could reduce the energy consumption and operating cost significantly. The results range from $0 \%$ to more than 50\% [2], [4], [5]; consequently, more thorough studies are required.

General trends and opportunities for operational optimization of district heating systems are discussed in [6], [7]. The operational optimization of (district scale) energy systems is often conceptualized in two steps, referred to as the unit commitment problem and the economic dispatch problem. The unit commitment problem aims at solving the discrete variables; the status (on/off) of each production unit is the main result. These problems belong to the class of integer or discrete optimization problems. Detailed load decisions for the active plants are taken in the economic dispatch problem. These problems belong to the class of continuous optimization problems. Detailed work on potential savings through sophisticated optimization methods at the district level is still lacking.

Studies have shown that the time taken and the associated project costs for implementing MPCs are an obstacle to their

\footnotetext{
${ }^{1}$ MPC is a control method based on repeated optimal control; while the optimization yields an optimal sequence spanning the optimization horizon, only the first sequence is applied to the system. 
practical use in todays buildings [2]. Studies have shown that between $55-70 \%$ of project costs are consumed by model creation and calibration [3], [8], which is consistent with findings from other areas, where MPC is applied [3].

Previous research has shown that acausal modelling approaches are well suited for rapid prototyping [9]. The model development time is significantly shorter compared to causal modelling approaches and it is easy to read, interpret, adopt, reuse and extend models.

The methods and tools demonstrated in this paper are suitable for overcoming difficulties with model development for optimization purposes. The work is based on Modelica, which is an open source modelling language that is designed for multi-domain analysis of dynamic systems. The ease of the modelling process is assured by the acausal, object-oriented character of the language.

\section{A. Contributions and Organization}

The main contributions of this paper are:

- A discussion of the state of the art of Modelica-based dynamic optimization.

- A scalability study of Modelica-based dynamic optimization of a building using a single shooting method implemented in the Toolchain for Automated Control and Optimization (TACO).

- A scalability study of Modelica-based dynamic optimization of a district heating system using direct collocation implemented in the OPTIMICA Compiler Toolkit (OCT); furthermore, the performance of the new version is compared with the older version used in [10].

In the literature, the comparison of various methods and algorithms is generally referred to as optimization benchmarking; the authors are aware that the comparison of different optimization methods and algorithms is a complicated task that necessitates many subtle considerations in order to yield a fair and unbiased evaluation [11].

Various motivations drive optimization benchmarking, such as (i) assisting the user to select the best algorithm or tools for specific tasks, (ii) showing the performance of new methods compared to existing ones, (iii) comparing new versions of software with earlier releases or (iv) comparing the performance of selected algorithms with different option settings [11].

It is not the goal of this paper to draw general conclusions about the scalability of Modelica-based dynamic optimization or the numerical methods examined in this paper, but to draw specific conclusions for TACO and OCT when applied to building and district models. Integer and mixed-integer optimization is beyond the scope of this problem; to the best of the authors' knowledge, there are no Modelica tools available that support mixed-integer optimization problems.

The rest of the paper is organised as follows. Section II gives a more detailed introduction to dynamic optimization and Modelica, the modelling language used in this work. The results of our study are then presented and discussed in section III and the conclusion is presented in section $\mathrm{V}$.

\section{BACKGROUND}

\section{A. Dynamic Optimization}

Dynamic optimization problems occur in the context of optimal control, MPC, parameter estimation or state estimation. The solution to the dynamic optimization problem is to find a trajectory that minimizes the objective function and satisfies the model equations and constraints. Dynamic optimization problems are applied in online and offline settings. In online applications, the solution time of the optimization problem is restricted by the operating conditions (often repeatedly). In offline applications, on the other hand, there are no restrictions on the solution time, as the optimization problem is solved before the system is set up and running [12].

Optimization methods can be classified as derivative-based and derivative-free methods. Derivative-based methods require derivative information of the model constraints and the objective function. Derivative-free methods do not require derivative information; for example, the optimization routine can integrate a detailed simulation program and evaluate the program as a black box.

The most widely used numerical methods for solving complex dynamic optimization problems are based on first-order necessary conditions for local optimality [13]. Methods can be categorized according to (i) when the system dynamics are discretized, and (ii) how they are discretized [12]. In the first point, a distinction can be made between direct and indirect methods. Indirect methods first establish the optimality conditions and then discretize the resulting differential equations to find a numerical solution. Direct methods first discretize the problem and then find an optimizer of the resulting Nonlinear Programming (NLP) problem. There are two different groups of methods to discretize the problem, namely shooting and collocation methods. Direct and indirect approaches can use the same method.

Optimization problems can be discretized manually and modelled in algebraic modelling languages (AMPL, GAMS, Pyomo). However, it is easier for the user to use tools designed specifically for dynamic optimization. The problem can then be formulated in its original form and the tool discretizes it. These tools can be based either on general purpose languages (C++, Matlab) or on component-based modelling (APMonitor, gPROMS, Modelica-based tools) [12]. The big advantage of component-based modelling is its ease of use.

Another important distinction for this paper is the distinction between block diagram modelling (also known as causal modelling) using imperative programming languages, and equation-based modelling (also known as acausal modelling). A detailed discussion of causal and acausal modelling of district energy systems can be found in [14]. Equation-based languages provide the following advantages for dynamic optimization: (i) they allow for automatic conversion of simulation models into optimization problems, which makes it more easily applicable for users and reduces the modelling effort; (ii) they can provide analytic expressions for gradients to be used by NLP solvers and (iii) the infinite dimensional problem can 
be automatically converted into a finite dimensional problem [15], [16].

\section{B. Modelica}

Modelica is an open source, acausal, equation-based, objectoriented and multi-domain modelling language. Originally, Modelica was developed for simulation-based analysis without direct support for optimization. In order to enable Modelicabased optimization, the language extension Optimica [15] was introduced, which among other functionalities, allows the formulation of objective functions and constraints. Based on Optimica, the language extension Dynamic Optimization Modelling Language was introduced [17]. Modelica-based dynamic optimization is currently supported by several open source tools including JModelica, OpenModelica or IDOS and commercial tools including OCT or TACO.

In recent years, Modelica-based dynamic optimization has been applied in different areas of energy systems such as buildings [18], district heating systems [7] or microgrids [19].

While these studies demonstrate the suitability of Modelicabased dynamic optimization in various areas, there is still a lack of scalability studies and performance comparisons with other methods and tools.

[16] compares the computing time to solve an optimal control problem of a building between a Modelica-based approach and a derivation-free simulation-based optimization (Nelder-Mead algorithm). The solution based on the collocation method was 2200 times faster.

The authors argue that it is not sufficient to test dynamic optimization approaches for small (test) examples; the implementations should also scale well to larger buildings and districts without running into convergence or optimization time problems.

Two different tools were used within this study:

- OPTIMICA Compiler Toolkit (OCT): The tool OCT [20] translate the Modelica and Optimica code to CasADi Interface, which has a flattened and symbolic representation of the model and optimization problem based on CasADi [21]. This representation is then propagated to the dynamic optimization algorithm implemented in JModelica.org [12]. This algorithm implements direct collocation to transcribe the problem into a NLP, which is then solved by IPOPT [22]. CasADi is used to compute first- and second-order sparse derivatives using algorithmic differentiation.

- Toolchain for Automated Control and Optimization (TACO): TACO [23] leverages the JModelica.org platform and CasADi to implement a custom NLP formulation that is tailored to building applications. The compact implementation leads to fewer optimization variables. The goal is that this approach leads to faster and more robust convergence of the optimization problem, while also supporting a larger part of the Modelica specification for optimization.

\section{USE CASES AND DYNAMIC OPTIMIZATION} APPROACHES

Two applications are presented to analyse the scalability of Modelica-based dynamic optimization. First, we demonstrate a use case for dynamic optimization of a multi-zone building using a VAV heating system. Second, we present a use case for dynamic optimization of a district heating system.

The general continuous optimization problem formulation is as follows:

$$
\begin{array}{cl}
\min _{x, y, u, p} & \phi\left(t_{f}, x\left(t_{f}\right)\right)+\int_{t_{0}}^{t_{f}} L(x(t), y(t), u(t)) \mathrm{d} t \\
\text { s.t. } & F(t, \dot{x}(t), x(t), y(t), u(t), p)=0, x\left(t_{0}\right)=x_{0} \\
& L \leq(\dot{x}(t), x(t), y(t), u(t), p) \leq U \\
& h_{e}(t, \dot{x}(t), x(t), y(t), u(t), p)=0 \\
& h_{i}(t, \dot{x}(t), x(t), y(t), u(t), p) \leq 0 \\
& H_{e}\left(t_{f}, x\left(t_{f}\right), y\left(t_{f}\right), p\right)=0 \\
& H_{i}\left(t_{f}, x\left(t_{f}\right), y\left(t_{f}\right), p\right) \leq 0 \\
& \forall t \in\left[t_{0}, t_{f}\right] .
\end{array}
$$

The system dynamics are described by the implicit, index-1 DAE in (1b), where $t \in\left[t_{0}, t_{f}\right]$ is time, $x$ is the state, $y$ is the algebraic variable, $u$ is the control variable and $p$ is the vector of parameters to be optimized. The objective function is the Bolza functional (1a), comprising the Mayer term $\phi$ and Lagrange integrand $L$. In the constraints, the simple bounds ((1c)) have been separated from the general path constraints ((1e) and (1d)), which can be on both equality and inequality form. Finally, there are the terminal equality and inequality constraints ((1f) and (1g)).

\section{A. Building}

The building model case is designed to demonstrate how the optimization computation time scales with the model complexity of a house. Therefore a series of models is designed that scales in a realistic, albeit simple way. The models consist of $n$ instances of the same submodel (called a single zone) that is illustrated in Figure 1. Each instance consists of a RectangularZoneTemplate from the Modelica IDEAS library (left) and a supply and return VAV (middle). The RectangularzoneTemplate consists of a zone model, four walls, a ceiling and a floor. Multiple instances of this base class are connected to a common fan and heater as illustrated for $n=8$ in Figure 2. Each zone is rotated by $360 i / n, i \in[1, n]$ degrees with respect to a reference direction such that the heat load profile of each building is different. For more details about the model we used we refer the reader to the IDEAS library [24]. Each zone instance has a single control variable that is used to control both the return and the supply VAV flow rates. Each VAV control variable is bounded between $[0,1]$. A control signal of 1 corresponds to a zone air change rate of 5 per hour. Air enters the heating device at the ambient temperature and is heated to the supply temperature set point of the model, which is another optimization variable. The supply temperature is upper bounded to $35^{\circ} \mathrm{C}$. Based on 
the resulting flow rates and temperatures, the heat flow rate of the heater is computed by the model, which is the objective function of this optimization.

In addition to these optimization variables and objective, we lower bound each zone temperature such that it is larger than $22{ }^{\circ} \mathrm{C}$. By lower bounding the heater heat flow rate to zero, we prevent cooling.

The resulting problem is parsed using the JModelica.org compiler, which is part of TACO for $n \in 1,2,4,8$, upon which TACO discretizes the model equations and compiles the model equations into an efficient $\mathrm{C}$ code using CasADi. The total horizon is 76 hours and we use a non-equidistant discretisation of 14 intervals. The optimization cases were run on a workstation with $32 \mathrm{~GB}$ RAM and six $3.6 \mathrm{GHz}$ cores.

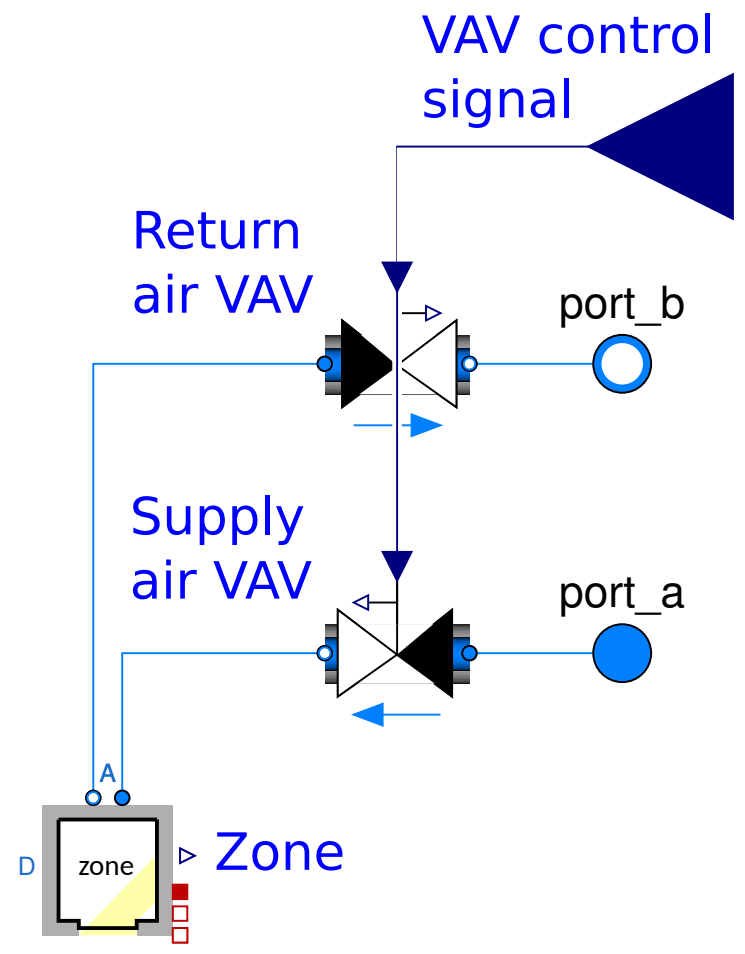

Fig. 1. The scheme of a single zone: consisting of a zone template model in the left (containing a zone, four walls, a ceiling and a floor), a supply VAV and a return VAV in the middle, and control input in the top right.

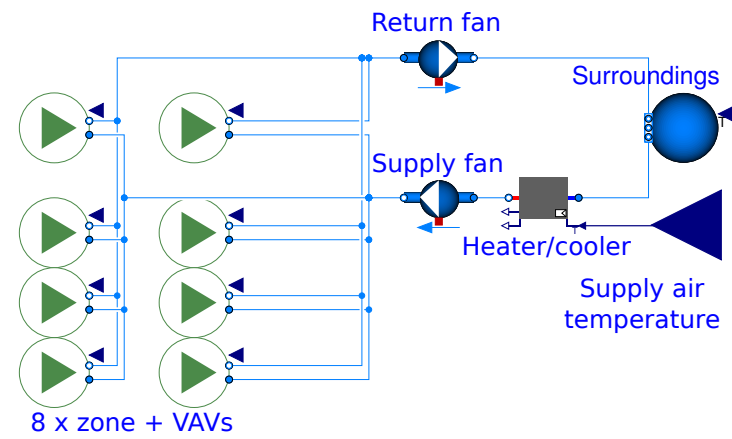

Fig. 2. The scheme of the complete model for $n=8$, consisting of 8 individual zones (See Figure 1), a supply fan, a return fan and a heater.

\section{B. District heating system}

The district heating case is designed to demonstrate how the optimization computation time scales with the remaining number of consumers after aggregating the original network. The case represents a thermal network in a planned city district in Graz, Austria that consists of one production unit, 16 consumers and a total length of about $4200 \mathrm{~m}$, see Figure 2. Details about the models and boundary conditions are described in [7]. The objective function is to minimize the supply water temperature and the pressure difference at the production unit; the load and pressure derivatives at the production unit are the degrees of freedom in the optimization formulation. The upper limit of the mass flow at the production unit was set to $65 \mathrm{~kg} / \mathrm{sec}$, which represents pump limitations. The lower limit of the customer supply temperature for all customers was set to $60^{\circ} \mathrm{C}$. The lower limit of the differential pressure for all customers was set to 0.5 bar.

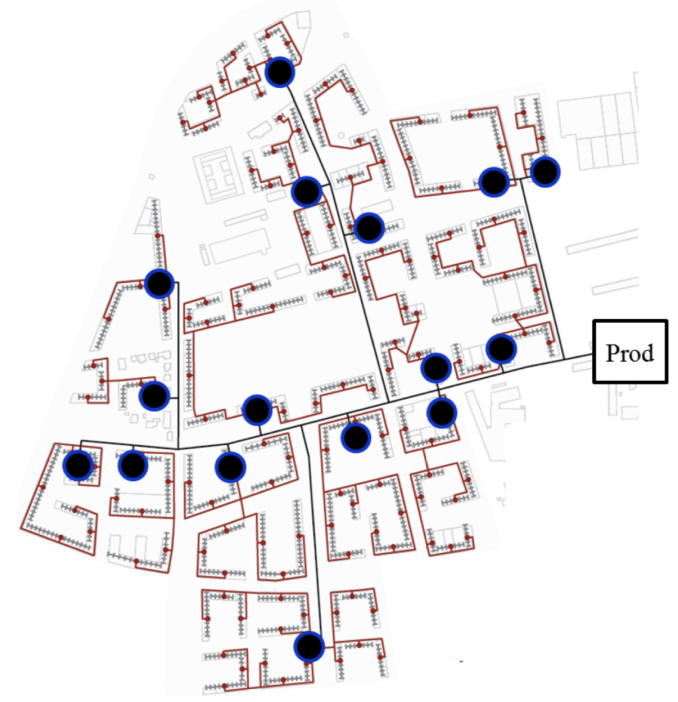

Fig. 3. The scheme of the district heating system. The production unit is seen on the right side; the black/blue circles represent the 16 consumers.

\section{RESULTS}

\section{A. Building}

The results of the building scalability test are summarized in Table I. The number of states scales linearly with $n$ since only the zone model contains state variables. Furthermore, the number of algebraic variables is included, which equals the "Number of time dependent variables" as outputted by Dymola 2019. The total number of NLP variables of the optimization problem that is solved by IPOPT is included in the third row of the table. Note that this number is much lower than the number of algebraic or state variables, taking into account that the problem is discretized into 14 segments. The translation time equals the total time required to read, translate and compile the optimization problem from the Modelica model code using TACO. The total time and number of iterations for solving the NLP using IPOPT are included in the sixth and seventh rows of 
Table I. Our optimizations start from zero initial guesses for all optimization variables by default. When instead warm starting the optimization from the solution of the previous hour, the number of iterations is reduced to about 7 for each value of $n$, reducing the IPOPT CPU time to $20 \mathrm{~ms}$ for $n=8$. Finally, the last row of Table I computes the CPU time per iteration, which reveals that the computation time of one iteration scales less than linearly with $n$ for the investigated values of $n$. This scaling is also illustrated in Figure 4.

TABLE I

SCALABILTY STUDY OF A MULTI-ZONE BUILDING MODEL USING SINGLE SHOOTING IMPLEMENTED IN TACO

\begin{tabular}{|l|r|r|r|r|}
\hline & \multicolumn{4}{|c|}{ Number of Zones } \\
\cline { 2 - 5 } & $\boldsymbol{1}$ & $\mathbf{2}$ & $\mathbf{4}$ & $\boldsymbol{8}$ \\
\hline Nr of states & 30 & 60 & 120 & 240 \\
Nr of algebraics & 498 & 782 & 1330 & 2746 \\
Nr of variables a.e. in NLP & 84 & 154 & 294 & 574 \\
Translation time [sec] & 33 & 44 & 71 & 197 \\
Iterations & 58 & 185 & 95 & 225 \\
IPOPT CPU time [sec] & 0.0362 & 0.248 & 0.18 & 0.63 \\
CPU time/iteration [msec] & 0.62 & 1.34 & 1.89 & 2.8 \\
\hline
\end{tabular}

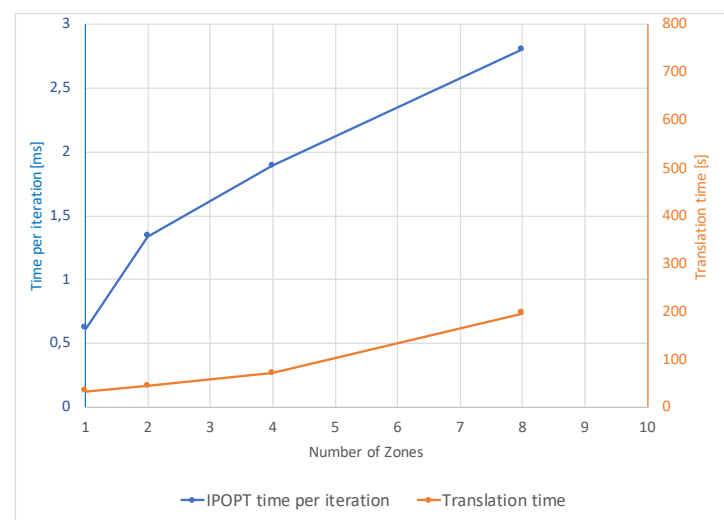

Fig. 4. Scaling of computation time per iteration and translation time with the number of zones for TACO.

\section{B. District heating system}

The results of the district heating test are summarized in Table II. The optimization cases were run on a laptop with $16 \mathrm{~GB}$ RAM and two $2.6 \mathrm{GHz}$ CPUs. The number of states as well as the number of algebraic variables scales linearly with the number of consumers. Note that the benchmarks differ slightly from Table I; the reason for this is a better comparability with [10]. OCT supports a block-lower triangular transformation of the DAE to identify algebraic variables that only depend affinely on the corresponding block variables; further variables can be eliminated by applying tearing to handle nonlinear dependencies. Thus, the majority of algebraic variables are eliminated prior to discretization, drastically reducing the size of the NLP. The remaining NLP contains between 20.000 and 60.000 variables. The number of iterations is almost constant for all cases. The wall-clock time equals the total time required (i) to create the network in Python NetworkX, (ii) to translate this network to Modelica code, (iii) to simulate the network, (iv) to aggregate the network depending on the requested number of remaining consumers, (v) to perform the dynamic optimization and (vi) to apply the optimal trajectories to the original network to verify the results. The framework is described in detail in [7]. The IPOPT total time and the wall-clock time scale linearly with the number of consumers. A comparison with the results in [10] shows that with the current setting (i) the total IPOPT time is between 5 and 6 times faster, (ii) the IPOPT CPU time is between 3.5 and 6.2 times faster and (iii) the wall-clock time is between 1.7 and 2.2 times faster. However, we must stress that these two studies were not carried out on the same computer nor on computers with identical computing power. Despite the differences in computing power, the authors assume that the newer version performs significantly better.

TABLE II

SCALABILITY STUDY OF A DISTRICT HEATING SYSTEMS USING DIRECT COLLOCATION IMPLEMENTED IN THE OCT.

\begin{tabular}{|l|r|r|r|r|}
\hline & \multicolumn{4}{|c|}{ Number of Consumer } \\
\cline { 2 - 5 } & $\mathbf{2}$ & $\mathbf{5}$ & $\mathbf{7}$ & $\mathbf{9}$ \\
\hline Nr of states & 12 & 22 & 30 & 38 \\
Nr of algebraics & 188 & 478 & 683 & 911 \\
Nr of variables a.e. in NLP & 18395 & 39079 & 45411 & 58845 \\
Iterations & 29 & 31 & 31 & 32 \\
IPOPT CPU time [sec] & 1.9 & 5.0 & 11.7 & 16.6 \\
IPOPT total time [sec] & 11.2 & 38.7 & 49.3 & 63.1 \\
Wall-clock time [sec] & 53.6 & 207.9 & 415.2 & 590.4 \\
\hline
\end{tabular}

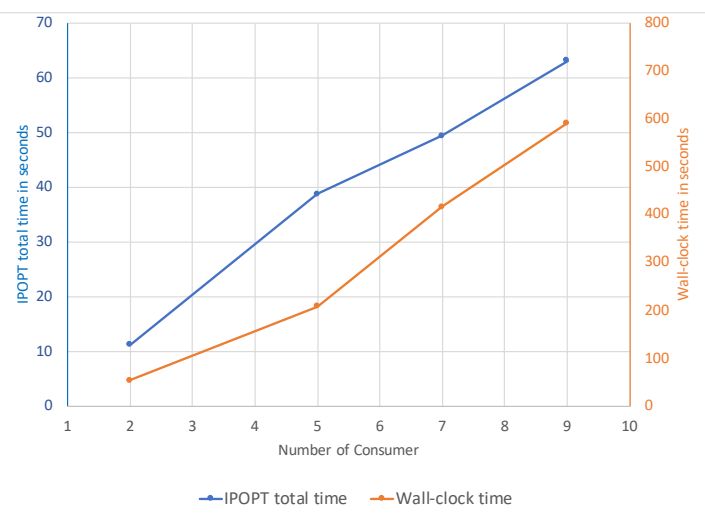

Fig. 5. Scaling of IPOPT total time and wall-clock time with the number of consumers in OCT.

\section{CONCLUSION}

The time required to create and calibrate models for sophisticated control strategies using state-of-the-art tools and methods hampers their practical use.

Due to its ease of the modelling process, Modelica is a promising language that is able to overcome this obstacle.

This paper presents a scalability study for Modelica-based dynamic optimization using two different tools, TACO and OCT. We presented two case studies: (i) dynamic optimization of multi-zone building models with a VAV heating system and 
(ii) dynamic optimization of a district heating system. Results of both case studies showed that linear scaling is possible for the tested range.

The definition of several use cases within district energy systems would be advisable in order to test the scalability of different approaches for large-scale dynamic optimization. Due to the potential for biased outcomes, we propose that these use cases should not be developed by tool vendors or specific modelling language experts. Furthermore, we propose that it would be better if the characterizations were done by domain experts who can be expected to be more neutral. DESTEST (developed in IBPSA Project 1) is a first step in this direction [25]; it includes the development of a validation test for district energy system.

Furthermore, many real world problems in district-energy systems require (mixed) integer formulations. State-of-theart Modelica tools do not support mixed-integer optimization problems; this poses a major challenge for future research and development.

\section{REFERENCES}

[1] F. Verrilli, S. Srinivasan, G. Gambino, M. Canelli, M. Himanka, C. Del Vecchio, M. Sasso, and L. Glielmo, "Model Predictive ControlBased Optimal Operations of District Heating System With Thermal Energy Storage and Flexible Loads," IEEE Transactions on Automation Science and Engineering, 2017.

[2] D. Sturzenegger, D. Gyalistras, M. Morari, and R. S. Smith, "Model Predictive Climate Control of a Swiss Office Building: Implementation, Results, and Cost-Benefit Analysis," IEEE Transactions on Control Systems Technology, 2016.

[3] P. Rockett and E. A. Hathway, "Model-predictive control for nondomestic buildings: a critical review and prospects Model-predictive control for non-domestic buildings: a critical review and prospects," Building Research \& Information, 2017.

[4] A. Afram, F. Janabi-Sharifi, A. S. Fung, and K. Raahemifar, "Artificial neural network (ann) based model predictive control $(\mathrm{mpc})$ and optimization of hvac systems: A state of the art review and case study of a residential hvac system," Energy and Buildings, 2017.

[5] J. Granderson, G. Lin, R. Singla, S. Fernandes, and S. Touzani, "Field evaluation of performance of hvac optimization system in commercial buildings," Energy and Buildings, 2018.

[6] A. Vandermeulen, B. van der Heijde, and L. Helsen, "Controlling district heating and cooling networks to unlock flexibility: A review," Energy, 2018.

[7] G. Schweiger, P.-o. Larsson, F. Magnusson, P. Lauenburg, and S. Velut, "District heating and cooling systems - Framework for Modelica-based simulation and dynamic optimization," Energy, 2017.

[8] J. Cigler, D. Gyalistras, J. Siroky, V.-N. Tiet, and L. Ferkl, "Beyond Theory: the Challenge of Implementing Model Predictive Control in Buildings," in Clima 2013 - 11th REHVA World Congress \& 8th International Conference on IAQVEC - "Energy Efficient, Smart and Healthy Buildings", 2013.

[9] G. Schweiger, H. Nilsson, J. Schoeggl, W. Birk, and A. Posch, "Modeling and simulation of large-scale Systems : a systematic comparison of modeling paradigms," Under Review, 2019.

[10] G. Schweiger, H. Runvik, F. Magnusson, P.-O. Larsson, and S. Velut, "Framework for dynamic optimization of district heating systems using Optimica Compiler Toolkit," in Proceedings of the 12th International Modelica Conference, 2017.

[11] V. Beiranvand, W. Hare, and Y. Lucet, "Best practices for comparing optimization algorithms," arXiv.org, 2017.

[12] F. Magnusson, "Numerical and Symbolic Methods for Dynamic Optimization Numerical and Symbolic Methods for Dynamic Optimization Fredrik Magnusson,” Ph.D. dissertation, 2016.

[13] L. T. Biegler, Nonlinear Programming: concepts, algorithms and applications to chemical processes, 2010.
[14] G. Schweiger, R. Heimrath, B. Falay, K. O'Donovan, P. Nageler, R. Pertschy, G. Engel, W. Streicher, and I. Leusbrock, "District energy systems: Modelling paradigms and general-purpose tools," Energy, 2018.

[15] J. Åkesson, K.-e. Årzén, M. Gäfvert, T. Bergdahl, and H. Tummescheit, "Modeling and Optimization with Optimica and JModelica.org Languages and Tools for Solving Large-Scale Dynamic Optimization Problems," Computers and Chemical Engineering, 2010.

[16] M. Wetter, M. Bonvini, and T. S. Nouidui, "Equation-based languages A new paradigm for building energy modeling, simulation and optimization," Energy \& Buildings, 2016.

[17] T. Tarnawski and R. Pytlak, "DOML - a Compiler Environment for Dynamic Optimization Supporting Multiple Solvers," in Proceedings of the 10th International ModelicaConference, 2014.

[18] R. De Coninck and L. Helsen, "Practical implementation and evaluation of model predictive control for an office building in Brussels," Energy and Buildings, 2016.

[19] J. Windahl, H. Runvik, V. Stephane, and S. Velut, "Platform for Microgrid Design and Operation Platform for Microgrid Design and Operation Platform for Microgrid Design and Operation," in Proceedings of the 13th International Modelica Conference, 2019.

[20] Modelon AB, "OPTIMICA Compiler Toolkit," 2019. [Online] Available: http://www.modelon.com/products/optimica-compiler-toolkit/

[21] J. Andersson, J. Åkessona, F. Casellad, and M. Diehl, "Integration of CasADi and JModelica.org," in Proceedings from the 8th International Modelica Conference, Technical Univeristy, Dresden, Germany, 2011.

[22] A. Wächter and L. T. Biegler, "On the implementation of an interiorpoint filter line-search algorithm for large-scale nonlinear programming," Mathematical Programming, vol. 106, no. 1, pp. 25-57, 2006.

[23] F. Jorissen, W. Boydens, and L. Helsen, "TACO, an automated toolchain for model predictive control of building systems: implementation and verification," Journal of Building Performance Simulation, 2019.

[24] R. Baetens, R. De Coninck, F. Jorissen, D. Picard, L. Helsen, and D. Saelens, "OpenIDEAS an Open Framework for Integrated District Energy Simulations," in BS2015, 14th Conference of International Building Performance Simulation Association, 2015.

[25] M. Wetter, C. V. Treeck, L. Helsen, D. Robinson, and G. Schweiger, "IBPSA Project 1 : BIM / GIS and Modelica framework for building and community energy system design and operation ongoing developments , lessons learned and challenges," in Sustainable Built Environment Conference 2019 (accepted manuscript), 2019. 